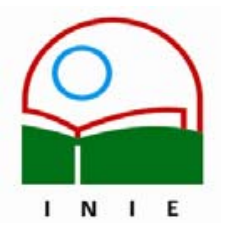

Universidad de Costa Rica

Facultad de Educación

Instituto de Investigación en Educación

ACTUALIDADES INVESTIGATIVAS EN EDUCACION

\title{
¿A DÓNDE JUEGAN NUESTROS NIÑOS Y NIÑAS?
}

\author{
Maureen Meneses Montero ${ }^{1}$ \\ María de los Ángeles Monge Alvarado ${ }^{2}$
}

Resumen: Un campo de juego es un área donde el individuo puede desarrollarse tanto física, social, intelectual $y$ emocionalmente, mediante programas culturales, comunales, escolares o actividades que él mismo quiera realizar.

El objetivo de este estudio, es dar a conocer qué es un campo de juegos, así como la normativa que rige estos espacios por parte de organismos internacionales, como la UNICEF y nacionales como el INVU. Se dan ejemplos del uso de estos espacios, tomando como referencia veinte de ellos, todos ubicados en el cantón de Montes de Oca en San José. La metodología es muy simple; se realiza un sondeo donde se observan principalmente condiciones y equipos que existen en los diferentes campos de juego, así como los administradores de los mismos.

Al observar a los niños y niñas jugar en una calle, donde transitan innumerables vehículos poniendo en peligro sus vidas, o cuando los vemos en un cuarto cerrado con cuatro paredes, cuestionamos el verdadero disfrute de jugar al aire libre. Aquí es cuando hay que cuestionarse sobre la importancia que conlleva un campo de juego y su valor pedagógico en las poblaciones infantiles.

Palabras clave: CAMPOS DE JUEGO, PLAYGROUND, NIÑOS Y NIÑAS

Abstract: a playground is an area where the individual is able to develop physically, socially, intellectually and emotionally; through cultural, community, or school programs; or through activities himself or herself may want to do.

The study's purpose is to determine what a playground is, as well as the regulations of national and international organizations (e.g. INVU and UNICEF, respectively) that define such spaces. Examples of the use of these spaces, making reference to twenty playgrounds located in San Pedro de Montes de Oca, San José. The method is very simple: an examination is made, where the main focus is towards equipment and conditions that exist in the different playgrounds and the managers of the spaces as well.

When the children are observed playing in the street, where innumerable vehicles are endangering their lives, or when we see them in closed, four-walled rooms, we ponder whether there is a true enjoyment in playing outdoors. Now is when we question the importance of playgrounds and their teaching value in the infantile population.

Key words: PLAYING FIELDS, PLAYGROUNDS, CHILDREN (BOYS AND GIRLS)

\footnotetext{
${ }^{1}$ Magister Scientae en Salud Pública, Licenciada en la Enseñanza de la Educación Física ambos por la Universidad de Costa Rica. Profesora de la Escuela de Educación Física y Deportes. Coordinadora de Actividad Deportiva, profesora de cursos de natación, métodos de enseñanza en la educación física, psicomotricidad en la educación inicial. También labora como profesora supervisora en las prácticas docentes y profesionales en la carrera de la enseñanza de la educación física. Actualmente funge como Subdirectora de la Escuela de Educación Física y Deportes. Profesora catedrática de la Universidad de Costa Rica.

Correo electrónico: menesesm@cariari.ucr.ac.cr

${ }^{2}$ Egresada del la Maestría Académica en Administración Pública, Licenciada en la Enseñanza de la Educación Física, labora como docente en la Escuela de Educación Física y Deportes. Miembro de la Comisión Nacional Movámonos Costa Rica, Miembro del Consejo Director del Programa de Salud Integral para los Cantones de Montes de Oca, Curridabat y La Unión. Profesora Asociada de la Universidad de Costa Rica.
}

Correo electrónico: mmonge@cariari.ucr.ac.cr

Artículo recibido: 16 de junio, 2004

Aprobado: 29 de noviembre, 2004 


\section{INTRODUCCIÓN}

En la declaración de los derechos de los niños y niñas, las Naciones Unidas afirman que: "el niño debe disfrutar plenamente de juegos y recreaciones... la sociedad y las autoridades públicas se esforzarán por promover el goce de este derecho" (UNICEF, 1983, p. 1). El cumplimiento del mismo recae directamente en la familia, la comunidad y el país en general, los cuales deben proporcionar actividades $y$, sobre todo, espacios lúdicos apropiados y saludables.

El juego, como actividad innata en el ser humano, es reforzado por todos los ambientes externos y las experiencias de movimiento que el niño va adquiriendo. Tolera todo el rango de habilidades de movimiento, las normas o reglas son autodefinidas. No hay victoria ni derrota, no hay comparación de uno mejor que otro; la actividad es espontánea e individual. Es en parte un reflejo del entorno que lo rodea; es una actividad necesaria, ya que contribuye al desarrollo social, emocional y físico del infante. Es la vida misma del niño y la niña.

Con el juego, los niños y las niñas aprenden acerca de sí mismos y de los demás, mediante su interacción con otros infantes y adultos, además los caracteriza y condicionan a que:

- aprendan cómo su comportamiento afecta a las otras personas y qué comportamiento es aceptable en su sociedad

a aprendan la oportunidad de expresar y vivir en forma genuina sus emociones y sentimientos

- favorezcan su desarrollo intelectual, al estar íntimamente ligados con todas las cosas físicas que le rodean

- desarrollen destrezas como correr, lanzar, apañar, columpiarse, entre otras que otorgan grandes beneficios al crecimiento físico

- se beneficien integralmente, identificándose más la necesidad de moverse para sentirse mejor, crecer y mejorar la salud mental, corporal y espiritual.

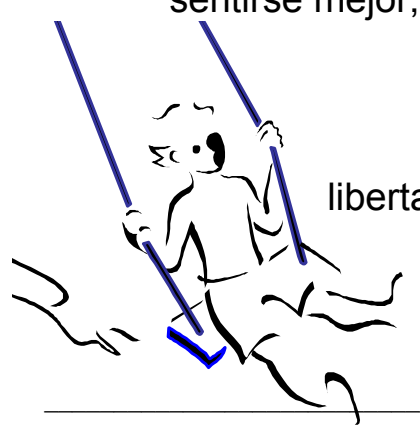

Flinchum (1988) considera que el juego abastece al niño y la niña de libertad para liberar la energía que tiene reprimida, fomentando así las habilidades interpersonales y le permite eventualmente, relacionarse y encontrar un lugar en el mundo social. 
A pesar de la necesidad que tiene el niño de jugar y de los efectos benéficos que posee, la sociedad, por intervención de los adultos, lo hace a un lado y no le dan el lugar que merece entre sus actividades, porque no brinda algún provecho económico y tangible.

Para otros representa una pérdida de tiempo y no creen en la función que ejerce en el niño y la niña. Los obligan a realizar actividades impropias para su edad, menospreciando los frutos que reciben a través de sus experiencias.

\section{El juego es tan necesario para el niño como la nutrición y el amor. Es el alimento de la parte emocional del ser.}

Los juegos deben corresponder a las capacidades de los niños y niñas, Becquer (s.f.) comenta que los juegos se deben aplicar ampliamente con fines pedagógicos y para el desarrollo multilateral de las generaciones crecientes; tienen un contenido rico y variado, en ellos se reflejan la realidad que rodea a la humanidad y, a la vez, representan una actividad de iniciativas y libertad propias, dirigida a vivencias alegres y activas.

El infante que juega siempre con ciertos objetos, posee percepciones y sensaciones muy diferentes sobre las cualidades de este objeto, con respecto a niños y niñas que no poseen esa oportunidad. Por ejemplo, de la forma, el color, el peso, la superficie, entre otros. Mientras más variada es la cantidad de juegos y los objetos utilizados, más percepciones y sensaciones variadas percibirá él y ella.

Frost, Bowers y Worthan, (1990, p. 19) presentan una serie de estudios que se resumen en el cuadro \#1 donde comprueban las atribuciones que tiene el juego en el bienestar de los niños y las niñas. 


\section{Cuadro \#1}

\section{Referencias de investigaciones y los beneficios del juego en los niños y niñas}

\begin{tabular}{|c|c|}
\hline Beneficios & \\
\hline - Promueve el desarrollo cognitivo & $\begin{array}{l}\text { Smith (1967), Piaget (1962), Salts y Brodie } \\
\text { (1982), Bruner,Jolly y Sylva (1967) }\end{array}$ \\
\hline el desarrollo & $\begin{array}{l}\text { Shure (1981), Ladd y Mize (1983), Harris } \\
\text { (1984) }\end{array}$ \\
\hline $\begin{array}{l}\text { Desarrolla las capacidades de } \\
\text { manipulación, arte imaginativo, } \\
\text { descubrimiento, razonamiento }\end{array}$ & Pepler y Ross (1981) \\
\hline - Des & $\begin{array}{l}\text { Smith (1968), Goodnow (1969), Dansky } \\
(1980)\end{array}$ \\
\hline - Mejo & $\begin{array}{l}\text { Sylva(1977), Smith y Dutton } \\
\text { Eisenberg y Harris (1984) }\end{array}$ \\
\hline$\triangle$ Des & Mc Cune-Nicolich (1981) Schirmer (1989) \\
\hline - Contr & $\begin{array}{l}\text { Klein (1932), Isaacs (1933), Axline (1947) } \\
\text { Erickson (1950) }\end{array}$ \\
\hline - Desarrollo de la & $\begin{array}{l}\text { Bennett }(1980), \\
\text { Staniford(1979) }\end{array}$ \\
\hline - Aum & $\begin{array}{l}\text { Smilansky (1968), Feitelson y Ross (1973), } \\
\text { Smith y Sydall (1978) }\end{array}$ \\
\hline$\triangle \mathrm{Au}$ & on y Ross (1973), Dansky (1980) \\
\hline - Aumenta el desarrollo de & $\begin{array}{l}\text { Vygotsky (1967), Lovinger (1974), Saltz, } \\
\text { Dixon y Johnson (1977) }\end{array}$ \\
\hline - Contribuye a la cooperación & Rosen (1974), Smith y Sidal \\
\hline
\end{tabular}

Frost, Bowers y Worthan, (1990) The state of American Preschool Playground Journal Physical

Education Recreatiom and Dance october 18-22

A partir de los beneficios anteriormente expuestos, encontramos que los campos de juego o play ground son una alternativa para que los niños y niñas se desarrollen armoniosamente, ya que en él convergen factores intrínsicos y extrínsecos a la personalidad de los seres humanos.

\section{¿CUAL ES EL ESPACIO DESTINADO A LOS NIÑOS Y NIÑAS PARA QUE JUEGUEN EN LA ACTUALIDAD?}

Años atrás, los niños, las niñas y jóvenes jugaban en las calles, potreros o en los jardines o patios de sus casas. Allí se jugaba quedó, escondido, salve la banca, salve el tarro, puritos, rayuelas, mirón-mirón, ambo-ambo, entre muchos otros más, o bien se subían a los árboles, y así se desarrollaban las habilidades y destrezas físicas de los niños, las niñas y jóvenes. 
Y hoy ¿qué pasó? Esos espacios desaparecieron. Un gran porcentaje del espacio de las ciudades está ocupado por la circulación de vehículos, las calles han dejado de ser un lugar donde se podía jugar. Es un espacio de peligro, donde tempranamente mueren niños y niñas víctimas de accidentes de tránsito.

Pero el precio del metro cuadrado en los residenciales se ha incrementado notablemente, por lo que las áreas verdes y espacios destinados al juego de los niños y las niñas se han reducido, no hay campo para jardines ni patios, casas juntas unas con otras han nacido al igual que los multifamiliares y condominios en forma vertical.

Con el fin de rescatar esa necesidad y derecho de todos los seres humanos, en Costa Rica hay una ley que regula las áreas de juego en las barriadas o complejos habitacionales, dictada por el Instituto Nacional de Vivienda y Urbanismo (INVU), en la cual se establece que todo urbanizador debe ceder gratuitamente un determinado porcentaje de terreno, dependiendo del total de metros cuadrados que ocupe la urbanización. Se establece así tanto un mínimo para desarrollos industriales y comerciales $(5 \%)$, como un mínimo para desarrollos turísticos (10\%). Se dice que el terreno donado deberá ser de igual o mayor calidad al resto. Finalmente, se aclara que las áreas de juegos infantiles, son requisitos de toda urbanización, en cuyas manos está la donación y mantenimiento de los equipos de play grounds. Por su parte, una organización comunal ayudará al establecimiento de gimnasios o canchas de cualquier índole. (INVU, 1973, p.13).

\section{¿QUE ES UN CAMPO DE JUEGO O PLAY GROUND}

Es un área recreativa que tiene sus orígenes, durante la Primera Guerra Mundial, ante la necesidad de adecuados programas de recreación por parte de las comunidades de la armada y de estaciones navales. Es así cómo mediante la Asociación de Servicio a la Comunidad del Campo de Guerra, se realizan actividades de recreación en pequeñas comunidades cercanas a los campos militares, para distraer tanto al personal militar y a civiles. Dado el beneficio

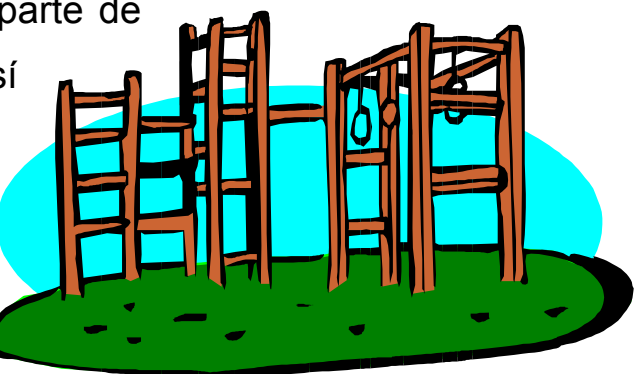
que obtuvieron con esos programas, se expandieron por todo el país y surge el movimiento de las áreas de recreación para las personas de todas las edades (Frost, 1994) 
Knudson (1984) considera el campo de juego como un centro de actividades para la comunidad, con un equipo adecuado para la experimentación y exploración del juego en los niños y niñas. Para Saez (2001, p. 1) "es el espacio de ocio que permiten a los pequeños divertirse, sin limitaciones, de una forma sana sin máquinas, sin riesgos y desarrollando al máximo su psicomotricidad". Salazar (1995, p. 49) los llama una fuente de experiencias que favorecen el desarrollo integral de los niños y niñas. Otros autores lo conceptúan como "un facilitador de eventos sociales y actividades deportivas, donde se lee, se discute, se escucha música, se realizan fiestas, se juega, las familias realizan picnics y donde la comunidad desarrolla sus eventos sociales y deportivos" (Friedlander y Lohmeyer, 1988, p. 70).

El campo de juego o play como se le conoce popularmente en el país, es un área que se les ofrece a los niños y las niñas para que realicen el juego que desean, según sus experiencias, sin la necesidad de programas organizados o dirigidos.

Tradicionalmente, la función de los campos de juego fue la de satisfacer la necesidad del juego a los niños y las niñas, mediante áreas al aire libre, con toboganes, columpios, sube y baja, cajas de arena, con programas de arte música, dramatización, deportes y hobbies. Tiempo después, esa función se ha ido cambiando, y se han modernizado sus estructuras y aparece el play ground aventura, el cual promueve el juego, la innovación, la exploración y la construcción con ayuda de túneles, laberintos, cuerdas, montículos de tierra, paredes y caminos para escalar y arrastrarse. (MacLean y Petterson, 1972)

\section{NORMAS EXISTENTES CON REPECTO A LOS CAMPOS DE JUEGO}

En Costa Rica, se dio un gran auge en el período presidencial Carazo Odio. En el despacho de la primera dama doña Estrella Zeledón de Carazo se apoya el proyecto de parques infantiles que se inicia en 1979, designado como el año internacional del niño. Se crearon 113 parques por todo el país, repartidos de la siguiente forma: 16 en Alajuela, 32 en Cartago, 9 en Guanacaste, 8 en Heredia, 4 en Limón, 13 en Puntarenas y 31 en San José, cuyos datos se encuentran descritos en informes del Instituto de Vivienda y Urbanismo (INVU,1984, p. 2).

En 1984, el INVU dictó una serie de normas que debían ser utilizadas en todo campo de juego, entre las cuales se estipula que el terreno donde se construya debe ser preferiblemente plano, en caso de presentar declives. Esto debe ser aprovechado 
creativamente en su diseño. Si la propiedad está muy cerca de vías de comunicación transitadas, debe tomarse en cuenta este aspecto para delimitar los límites del mismo.

En los campos de juego, se estima conveniente que el equipo: hamacas, columpios, toboganes, etc., sean de formas y colores llamativos, y debe cuidarse la ubicación de estos de manera que puedan ser utilizados seguramente, y con el espacio suficiente para evitar accidentes.

Las superficies pueden combinarse con zonas verdes y "planchés" de cemento, los cuales se pretende que sirvan para dibujar rayuelas $u$ otros juegos de tipo tradicional. Las zonas verdes tienen como objetivo estimular el contacto con la naturaleza.

Estas áreas son visitadas por niños y niñas de diferentes edades, por lo que se estima que las hamacas, columpios, toboganes, túneles, puentes, pasamanos deban tener diferentes tamaños. Cerca de ellos se colocarán bancas para los responsables del cuidado de los infantes.

Si el material de que está hecho el equipo, es de madera, ésta debe ser "curada" para que no se pudra y el equipo construido con hierro debe ser tratado para evitar que se oxide y dañe a los visitantes del parque.

Se recomienda que estos espacios sean amplios, no reducidos para que los niños y las niñas puedan desenvolverse según su edad, en sus propios espacios de acción; lógicamente deben contar con techos o resguardos contra la lluvia y el viento.

Las plantas utilizadas para la decoración de los mismos o para delimitar los espacios, no pueden ser venenosas o que produzcan alergias a los visitantes. Sus drenajes deben ser eficientes y limpios, para que el paso de las aguas sea fluido y evitar el empozamiento que pudiera servir como criadero del mosquito que infecta a los seres humanos del dengue.

Es importante también contar con basureros suficientes y al alcance de los visitantes dentro de las regulaciones. Así se promueve el ornato y aseo dentro del parque y la comunidad. 
Al aplicarse estas normas la institución pretende dar un marco legal y operativo a la construcción de los parques infantiles, y poder estandarizar todo el equipo que se construya en ellos.

La UNICEF norma desde 1983 la construcción de parques infantiles en el nivel internacional. Algunas de las recomendaciones se resumen a continuación:

- Tomar en cuenta el tamaño del terreno para seleccionar adecuadamente los juegos y su distribución.

- El parque debe ser construido cerca de viviendas, escuelas, mercados y centros de salud, entre otros para que su utilidad sea mayor y más segura.

- El parque debe estar alejado de calles o avenidas de mucho tráfico.

- Las irregularidades del terreno deben ser tomados en cuenta en el diseño de los juegos y hacer del parque un lugar importante.

- Para la construcción, mantenimiento y cuidado y mejoramiento de los parques, los comités y autoridades del lugar deben organizarse en grupos de trabajo, fortaleciendo y asegurando este espacio.

- No se debe cercar el parque con alambre de púas o materiales cortantes o punzantes, además se debe tener cuidado de eliminar las plantas venenosas.

- El parque debe estar alejado de gasolineras, barrancos, basureros, bares, fábricas, pozos, zanjas, aguas negras, alambres eléctricos alcanzables.

- En los parques no deben existir cosas y objetos puntiagudos, materiales inflamables, cortantes, que representen peligro o que puedan causar daño.

- Las corrientes de aguas sucias, hoyos, depósitos naturales y charcos que sirven de criaderos de zancudos deben ser eliminados.

- Se aprovecha para la construcción de los juegos, equipos y materiales: troncos, aros, cables, correas, cadenas, tubos de desagüe, varillas, llantas, tablas, tableros, caballetes, escaleras, tubos, caños, carros, carretas viejas, árboles, argollas, reglas, cajas, barras, vagones de tren, fosos de arena, piedras, bloques, adobes, cercas, mallas, barriles o estañones. Los materiales citados anteriormente se pueden adaptar, arreglar, pintarse de tal modo que sean seguros, vistosos, atractivos y protegidos ante las inclemencias del tiempo y del ser humano. 


\section{EL ROL DEL ADULTO EN EL CAMPO DE JUEGO}

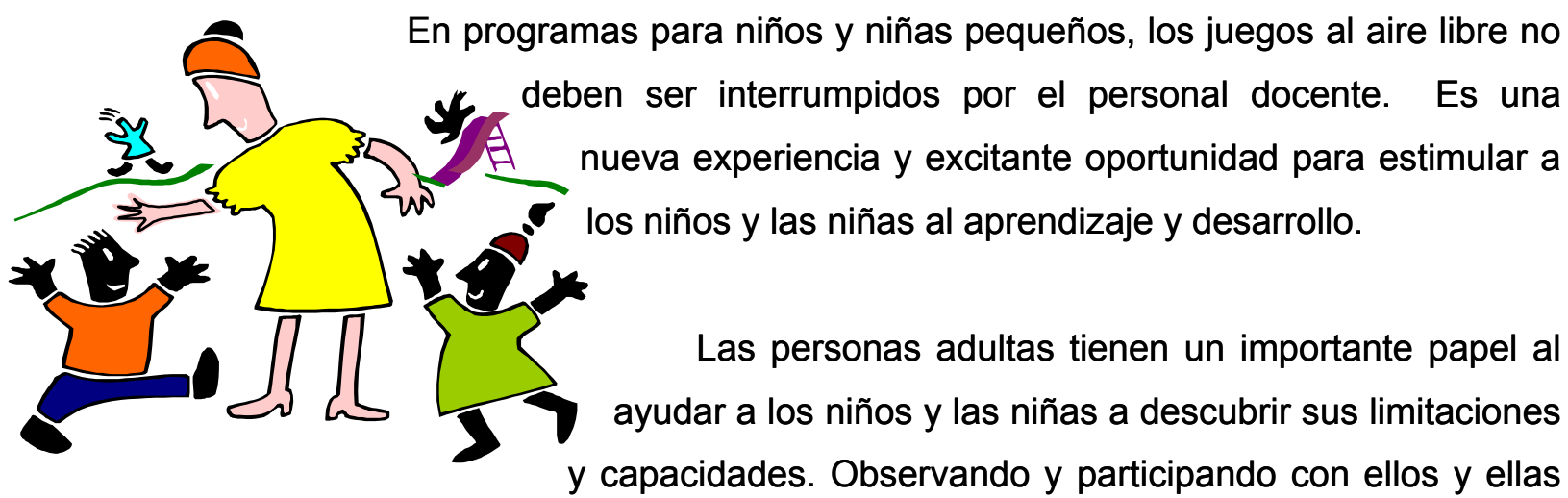
en los juegos al aire libre, el personal docente puede identificar las diferencias individuales en sus habilidades e intereses. Se pueden usar estas observaciones para planear ricas y variadas experiencias de juego, las cuales permitan a los niños y las niñas desafiarse a sí mismos.

\section{EL ROL DE LA COMUNIDAD}

La comunidad es el reflejo de la organización y mantenimiento de los campos de juego. La mayoría pertenecen a municipalidades; sin embargo, se mantienen la mayoría del tiempo sin la supervisión necesaria para su funcionamiento. Es aquí cuando la labor comunal se hace patente y comparte su responsabilidad.

Para ilustrar el rol de la comunidad se realizó un sondeo en el cantón de Montes de Oca; se visitaron 20 campos de juego. Algunos de ellos llamados parques infantiles; sus dimensiones varían de $100 \mathrm{mts}^{2}$ a 33 manzanas. Básicamente se observaron las condiciones y los equipos con que cuentan. Se resume la información en el cuadro \#2.

\section{CUADRO \#2}

Resumen de campos de juego del cantón de Montes de Oca.

\begin{tabular}{|l|c|l|}
\hline \multicolumn{1}{|c|}{ Distritos } & $\begin{array}{l}\text { No. Campos } \\
\text { de juego }\end{array}$ & \multicolumn{1}{|c|}{ Condiciones } \\
\hline $\begin{array}{l}\text { Cedros, San Rafael, } \\
\text { Mercedes, San } \\
\text { Pedro, Sabanilla, }\end{array}$ & 20 & $\begin{array}{l}\text { En su mayoría existen hamacas, toboganes, } \\
\text { sube y baja, caballito, argollas, pasamanos en } \\
\text { condiciones muy precarias. } \\
\text { Algunos de ellos (5) cuentan además con áreas } \\
\text { verdes, y espacios destinados a canchas de } \\
\text { baloncesto y papi fútbol. lgualmente las } \\
\text { condiciones no son las más adecuadas }\end{array}$ \\
\hline
\end{tabular}


Se hace importante rescatar que la mayoría de estos lugares son mantenidos en general por los vecinos, ya que a nivel municipal, el apoyo básicamente se centra en recoger la basura y mandar equipos de mantenimiento a cortar el zacate. La mayoría de encargados de estas zonas son vecinos voluntarios.

El área de juegos más grande del cantón se encuentra en el Parque del Este, espacio perteneciente al Instituto Costarricense de Deportes y Recreación (ICODER) que cuenta con aproximadamente 33 manzanas, el cual se mantiene en buenas condiciones en general.

Todos los campos de juegos visitados tienen acceso abierto: algunos de ellos poseen cercas alrededor, lo que ha facilitado cerrarlos durante las noches y evitar destrozos por parte de delincuentes. Otro punto importante es que a pesar de que la ley del INVU obliga a la creación de estos espacios en los residenciales, el mantenimiento no le corresponde, lo que obliga a las municipalidades a dar un servicio que no resulta eficaz.

\section{LA SEGURIDAD EN LOS CAMPOS DE JUEGO}

Para Frost (1994) la clave para obtener la mayor seguridad se basa en cuatro variables, que consisten en contar con un equipo seguro, seleccionar los fabricantes objetivamente por la calidad de su mercadería, que lleven pólizas de seguridad y además que ofrezcan expertos en el diseño y consultores en el diseño del equipo y en el desarrollo de los niños y las niñas. Así mismo Hudson, Thompson y Mack (1997) han señalado que la edad apropiada, superficie, supervisión y mantenimiento son los cuatro elementos que interactúan para crear ambientes seguros en los campos de juego y citan a Thompson y Hudson (1996, p. 33) como se ilustra en la figura \#1: 


\section{Figura \#1}

Elementos para crear ambientes seguros en campos de juego.
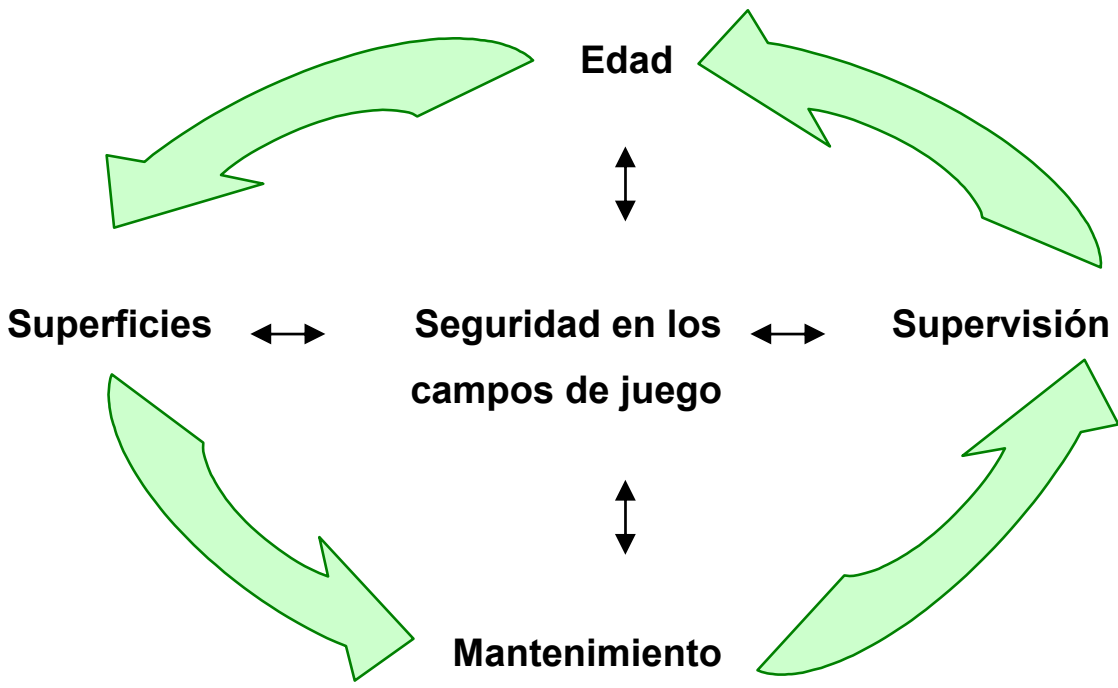

Tomado de: Hudson,S; Thompson,D; Mack,M (1997) Are we safe yet? A twenty-five year look at playground safety. Journal Physical Education Recreation and Dance. Vol 68 N 8,32-34

Se debe entender que el equipo debe corresponder al tamaño, según la edad y las características físicas y mentales de los niños y niñas.

Es recomendable que exista un área solamente para niños y niñas de 2 a 5 años y otra para los de 6 a 12. Esta separación es necesaria para la seguridad de los niños y niñas pequeños. Para los infantes que empiezan a caminar, también es necesario un espacio, con equipo y zonas diseñadas que obedezcan a las etapas del desarrollo.

La superficie sobre la que va el equipo debe ser adecuada, elástica y resistente, con espacio suficiente entre uno y otro equipo, labor que debe ser llevada a cabo por profesionales en la ingeniería. Se menciona que entre el 60 y el $70 \%$ de todos los accidentes ocurridos en estos lugares y el $90 \%$ de los más serios, resultan debido a las caídas de los niños y niñas en las superficies (Knudson, 1995, p. 86).

El autor citado anteriormente, menciona una situación ocurrida en las décadas pasadas en los Estados Unidos de Norte América, según como se describe es lo que actualmente está pasando en Costa Rica. Con el tiempo, el terreno de los campos de juego, fue compactado en roca sólida por los pies de los niños y niñas, mientras que el equipo está 
deteriorado. Para compensar los agujeros llenos con agua y lodo localizados en áreas de abundante tránsito de personas, la pavimentación con asfalto llegó a ser el material escogido para las superficies del piso. Estos patrones, superficies duras y equipo deteriorado asociados con el descuido y no exigente mantenimiento, es el escenario propicio para el crecimiento de una serie de accidentes por parte de los niños y las niñas.

Al igual que Frost (1994) y Sawyers (1994) se hace referencia a que la mayoría de accidentes que ocurren en los campos se deben a las caídas que sufren desde los columpios, toboganes y otros.

La supervisión en el uso de los aparatos es de gran ayuda para disminuir el riesgo de accidentes. Estas personas como bien lo dicen Hudson, Thompson y Mack (1997) deben ser entrenados en su función.

Y por último, se encuentra el mantenimiento de la instalación y del equipo, variables que si se desarrollan eficiente y eficazmente proveerán un ambiente seguro a sus ocupantes. Los autores concuerdan en que este personal debe ser capacitado en instalar y mantener tanto el campo como los diferentes equipos existentes en el área.

\section{LOS ADMINISTRADORES DE LOS CAMPOS DE JUEGO}

Los administradores de los campos de juegos, llámense municipalidades, comités cantonales o comunales, juntas administradoras, teóricamente son los responsables del uso que se les da a estas instalaciones y son los encargados de dar respuesta a todas las quejas hechas por parte de los usuarios o de las personas comprometidas en el cuido de los niños y las niñas asistentes.

El mantenimiento y la supervisión deben ser responsabilidad de la organización a la que está a cargo el campo de juego.

Los campos de juegos deben ser revisados periódicamente. Se debe llevar a cabo un programa de seguridad basado en descubrimientos y recomendaciones que se deben realizar prontamente. Todo accidente que se dé, debe apuntarse en un "cuaderno", con la descripción precisa de lo ocurrido, fecha, hora, testigos, parte médico y policial, así como otros elementos adicionales que el encargado crea conveniente. 
Además éstos deben hacer cumplir los reglamentos que se tienen para su uso; muchos de los accidentes que ocurren, a parte del estado del equipo, se deben a que tanto los responsables, como los mismos infantes utilizan el equipo que es para niños y niñas mayores.

El administrador debe colocar señales de prevención a la entrada del campo, así como su reglamento. Entre éstas se encuentran: el rango de la edad para el cual ese espacio está destinado, los niños y las niñas deben ser supervisados por adultos todo el tiempo o para resaltar algo inusual en el ambiente, pero que en ese momento, está apareciendo. Estas referencias refuerzan el cuidado que se debe tener.

Esta persona debe permanecer en el campo de juego, mientras esté abierto y servir de supervisor de los juegos que realizan los niños y niñas, ya que para evitar el aburrimiento, entonces incrementan los retos que el equipo les proporciona. Sawyer (1994) cuenta ejemplos de las acrobacias que realizan como mecerse con un pie en una hamaca, pasarse de una hamaca a la otra de pie, cuando la hamaca está en el punto más alto, saltar hacia la tierra, enrollar la cadenas y otros.

\section{CONCLUSIONES}

A pesar de la importancia de los campos de juego en el desarrollo integral de los niños y las niñas, estos espacios no son prioritarios en la mayoría de las comunidades. Algunos miembros de ellas se encargan voluntariamente, sin contar con los materiales y presupuestos necesarios para todas las obras de mantenimiento. Cuando las escuelas y las casas de habitación no cuentan con los patios de recreo necesarios para el esparcimiento de la niñez, una opción son los parques o campos de juego; sin embargo, se convierten contrariamente en zonas peligrosas, ya que las hamacas, toboganes y otros están semidestruidos o en condiciones que no permiten el claro desarrollo de la motricidad infantil.

El movimiento es esencial en el ser humano; explotar este componente es responsabilidad de las personas adultas, por lo que programas educativos y la comunidad deben velar por un claro desarrollo de los niños y las niñas y, a la larga, los campos de juego se convierten en una alternativa accesible y barata, ya que en el caso de Costa Rica, todas las urbanizaciones, barrios, deben contar con este espacio. 


\section{REFERENCIAS}

Alpírez, F. (1981). Campos de juego y su influencia en el desarrollo del niño en la etapa preescolar. Trabajo mimeografiado, Universidad Nacional. Costa Rica.

Bécquer. (s.f.). Educación física en Preescolar. Folleto mimeografiado, Cuba: INDER.

Bowers, L. (1988). Children need playground, but playground need help Journal Physical Education Recreation and Dance. Vol 58 (9) 47-51.

Flinchum, B. (1988). Early childhood movement programs. Preparing teachers for tomorrow. Journal Physical Education Recreation and Dance. Vol 59 (7) 62-64.

Friedlander, R. y Lohmeyer, R. (1988). Games and Sports Task for Young Children. Journal Physical Education Recreation and Dance, Vol 58 (9)70-72.

Frost, J. (1994). Prevention playground injuries and litigation. Parsk and Recreation. Abril 53-60.

Frost, J.; Bowers, L.; Worthan, S. (1990). The state of American Preschool Playground. Journal Physical Education Recreation and Dance. Vol 62 (10)18-22 .

Hudson, S.; Thompson, D.; Mack, M. (1997). Are we safe yet? A twenty-five year look at playground safety. Journal Physical Education Recreation and Dance. Vol $68 \mathrm{~N}^{\circ}$ 8,32-34.

Instituto Nacional Vivienda y Urbanismo (INVU). (1973). Reglamento para el control nacional de fraccionamientos y urbanizaciones. Costa Rica: Imprenta Nacional.

Instituto Nacional Vivienda y Urbanismo (INVU). (1984). Juegos Infantiles. Costa Rica: Imprenta Nacional.

Knudson, D. (1995). Outdoor recreation. USA: McMillan Publishing Company.

Marín, I., Penón, S. (1997). Los parques infantiles de juego. La factoría No 3.

Recuperado el 28 de abril del 2001, de

http://www.lafactoriaweb.com/articulos/marpen3.htm

Mc Lean, C.; Petterson, D. (1972). Recreation and leisure: The changin scene. 3a ed. California, USA: Wadsworth Publishing.

Saez, C. (2001). Parques infantiles, el predeporte infantil. Recuperado el 28 de abril del 2001, de http://www.temueves.com

Salazar, C. (1995). Seguridad en los campos o parques de juego. Revista Costarricense de Salud Pública. 49-54.

Sawyers, J. (1994). The preschool playground: developing skills through outdoor play. Journal Physical Education Recreation and Dance. (8) 31-33. 
UNICEF. (1983). Jugando y creciendo. Parques infantiles, ideas para su construcción. Guatemala: Editorial Piedra Santa. 